\title{
Secondary User Power Saving in Overlay Cognitive Radio Networks
}

\author{
Ahmed Masri \\ An-Najah National University \\ Nablus-Palestine
}

\author{
Saed Tarapiah \\ An-Najah National University \\ Nablus-Palestine
}

\author{
Yousef Dama \\ An-Najah National University \\ Nablus-Palestine
}

\begin{abstract}
The secondary user power saving in the overlay based Hybrid Automatic Repeat request cognitive radio has been investigated. The effect of the power splitting ratio on the secondary transmissions has been studied and a simple power saving technique for secondary users is proposed. Extensive simulation runs have been carried out to validate our results.
\end{abstract}

\section{General Terms:}

Power Saving

\section{Keywords:}

Cognitive Radio, Overlay, OFDM-CP

\section{INTRODUCTION}

Overlay access paradigm allows concurrent secondary and primary transmissions, through splitting the secondary power for secondary communication part and the remainder of the power to assist (relay) primary transmission [9, 3]. By careful choice of the power splitting ratio, the increase in a Primary User (PU) Signal to Interference plus Noise Ratio (SINR) due to the assistance from secondary relaying is exactly offset by the decrease in the PU's SINR due to the interference caused by the remainder of the secondary transmit power that is used for secondary communication.

The work in [2] shows that overlay access paradigm can outperform other paradigms (namely, the underlay and the interweave [1] 9]) in terms of capacity. However, overlay paradigm suffers from several practical limitations, due to the prior knowledge requirements on the secondary transmitter, i.e., a prior knowledge about the PU's message and the full Channel State Information (CSI). These limitations have to be fulfilled to guarantee seamless secondary co-existence within the primary system.

Tannious and Nosratinia [7] proposed a modified overlay cognitive radio model, in which the primary utilizes a Hybrid Automatic Repeat request (HARQ) protocol. According to their proposal the secondary link is only active during primary retransmissions. Thus, the secondary pair is able to obtain the prior knowledge about the PU's message through listening to the PU's first transmissions, as shown in Figure 1 Moreover, the authors in [7] suggested two protocols based on HARQ (namely, the Split and the Power control). The former protocol requires either full or partial non-causal knowledge of CSI at the secondary transmitter, something that is hard to achieve in practice. While the latter protocol, does not require any CSI knowledge at the transmitter, but considerably penalizes the achievable secondary throughput and still it has some co-existence penalty at the primary owing to the secondary transmission.

The recent work about overlay cognitive radio access paradigm [9, 3, 2, 1, 7, 8], focus on how to achieve the prior knowledge requirements on the secondary transmitter while protecting the primary system. However, this effort does not study neither the Secondary Users (SUs) power saving nor the power splitting ratio values and their effect on the secondary transmission. The Power control protocol in [7], suggested the possibility for SUs to save power through keeping silent, however no further details is given. Accordingly, the contribution of this paper is twofold: (i) we enhanced the HARQ Split protocol and we studied the effect of the power splitting ratio on secondary transmissions, (ii) we investigated the SUs power saving and we proposed a simple power saving technique that will allow SUs to save a significant amount of power.

The rest of the paper is organized as follows. Description of the system model in section 2 Then in section 3 , we investigate the power splitting ratio and the SUs power saving. We present the performance of our enhanced Split protocol with Power Saving (Split$P S$ ) and compare it to the conventional Split protocol in section 4 Finally, in section 5 we draw some conclusions.

\section{SYSTEM MODEL}

We consider a network scenario with four nodes, for the sack of simplicity; primary transmitter and receiver and secondary transmitter and receiver as in Figure 2 The fading between the nodes is of the flat quasi-static Rayleigh type, where a per block-fading channel model is used with effective channel gains $\left|h_{i, j}\right|^{2}$ are exponentially distributed. We assume that SU applies no rate control and uses a fixed transmission power $P_{s}$. Moreover, a feedback channel is exist between the secondary pair. Furthermore, the link outage is defined as in [4], where it is the event that the mutual information across the channel is less than the attempted rate $R$ and 


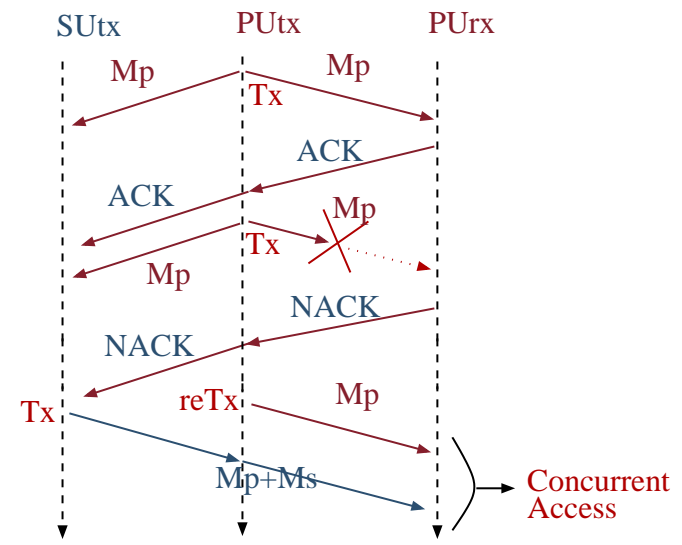

Fig. 1. Message transmission using HARQ protocol is presented between a primary pair $\left(P U_{t x}, P U_{r x}\right)$, while a $\mathrm{SU}\left(S U_{t x}\right)$ is listening and trying to access the primary spectrum concurrently with the primary retransmission, using an overlay access scheme. Moreover, all SUs and PUs are within same transmission range and $\left(M_{p}, M_{s}\right)$ are the PU's and SU's messages, respectively.

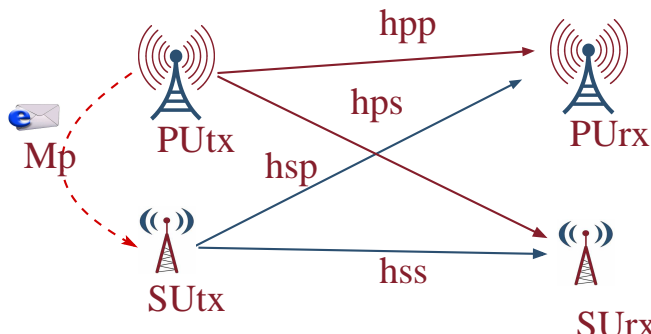

$\mathrm{Mp}$

-.. : Through HARQ

Fig. 2. Network scenario with a couple of primary and secondary pairs is presented. All users are within the same transmission range and the dotted line indicates a priori knowledge of the PU's message $M_{p}$ at the SU transmitter $S U_{t x}$.

the link outage probability between a pair of users $(i, j)$ is given by:

$$
\mathbb{P}_{\text {out }}=\mathbb{P}\left\{\log _{2}\left(1+\left|h_{i, j}\right|^{2} P_{i}\right) \leq R_{i}\right\}
$$

We adopt the overlay based HARQ Split protocol in |7| for obtaining the prior knowledge about the PU's message, due to its ability of full elimination of co-existence penalty at primary system, even under concurrent secondary transmitters.

In Split protocol, each secondary transmitter will compensate for its interference by relaying the primary message. In addition, SU can acquire through overhearing the ACK/NACK signals (i) one bit of information about the magnitude square of the primary channel gain, i.e., if it is less or more than a certain threshold $\left|h_{p, p}\right|^{2}<\left(2^{R_{p}}-1\right) / P_{p}$, given the knowledge of PU's power $P_{p}$ and rate $R_{p}$, (ii) an estimate of the $\left|h_{s, p}\right|^{2}$ (the channels are assumed to be reciprocal).
We contribute to the Split protocol by overcoming its main drawback, through relaxing its non practical assumption about the non-casual prior knowledge of the primary's CSI $\left(h_{p, p}\right)$ on the secondary transmitter, that is needed to allow coherent sum on the primary receiver. The non-casual prior knowledge is no more required on the secondary transmitter by assuming the use of the Orthogonal Frequency Division Multiplexing with Cyclic Prefix (OFDM-CP) on the primary transmitter.

Our assumption will allow the secondary signal (relay part) and the primary signal to sum coherently on the primary receiver, without the need of exact knowledge about $h_{p, p}$. This is true as long as we maintain a relative delay between the secondary relaying signal and primary signal that does not exceed the duration of the cyclic prefix of the primary transmitter signal [5]. Without loss of generality, we assume a perfect synchronization between the primary and secondary transmitters.

Finally, our analysis are a long the lines of the analysis in [7] and we compute the power splitting ratio $\alpha \in[0,1]$ as in the Split protocol, in which it has to satisfy the following condition:

$$
\begin{aligned}
& \log _{2}\left(1+\frac{\left(\left|h_{p, p}\right| \sqrt{P_{p}}+\left|h_{s, p}\right| \sqrt{\alpha P_{s}}\right)^{2}}{1+\left|h_{s, p}\right|^{2}(1-\alpha) P_{s}}\right) \\
& =\log _{2}\left(1+\left|h_{p, p}\right|^{2} P_{p}\right)
\end{aligned}
$$

The condition in Equation (2) is to guarantee that the PU throughput during the coexistence case with the $\mathrm{SU}$ is equal to the PU throughput in the case of SU absence.

Next, we study the effect of the power splitting ratio values on the secondary transmission and we propose a simple technique to allow secondary users to save power.

\section{THE POWER SPLITTING RATIO AND SECONDARY USERS POWER SAVING}

As discussed in the previous section, the power splitting ratio computation takes into account the PU protection only, without any consideration about secondary system. Accordingly, in this section we study the power splitting ratio and its effect on the secondary system, through simulating two network scenarios, (namely, Close and Far).

In Close scenario, the SUs transmitter and receiver are relatively close from each other and from the PU transmitter, while they are far from the PU receiver, i.e., best case scenario. On contrary, in the Far scenario the SUs transmitter and receiver are relatively far away from each other and from the PU transmitter, while the secondary transmitter is close to the PU receiver, i.e., worst case scenario. Accordingly, we reflect the proximity of the secondary pair to the primary transmitter compared to the distance between PUs pair by setting the means of the effective channel gains to be equal to $\left\{\mu_{p, p}=\mu_{s, p}=1, \quad \mu_{p, s}=\mu_{s, s}=4\right\}$ and $\left\{\mu_{p, p}=\mu_{p, s}=\mu_{s, s}=1, \quad \mu_{s, p}=4\right\}$ for the Close and Far scenarios, respectively.

Figure 3 shows the Probability Density Function (PDF) for both the power splitting ratio and the corresponding secondary link outage probability, while using three different SU power levels $\left(P_{s}=10,15\right.$ and $\left.20 \mathrm{~dB}\right)$. In Figure $3 \mathrm{~d}$, we notice the high sec- 


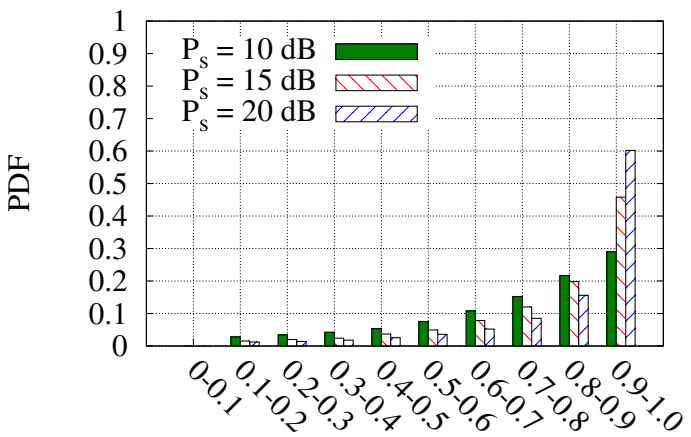

Power splitting ratio $(\alpha)$

(a) Close scenario

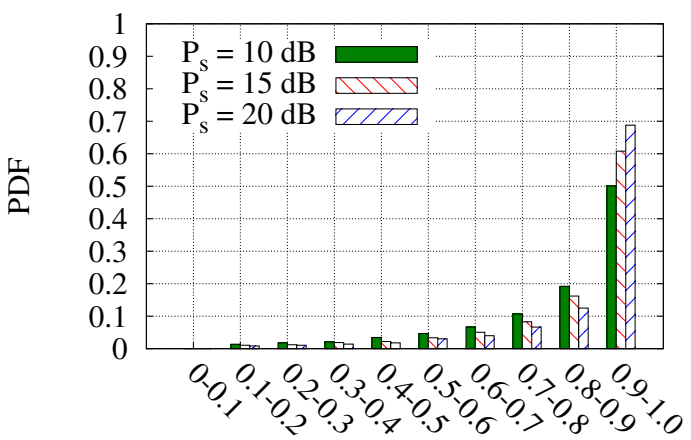

Power splitting ratio $(\alpha)$

(c) Far scenario

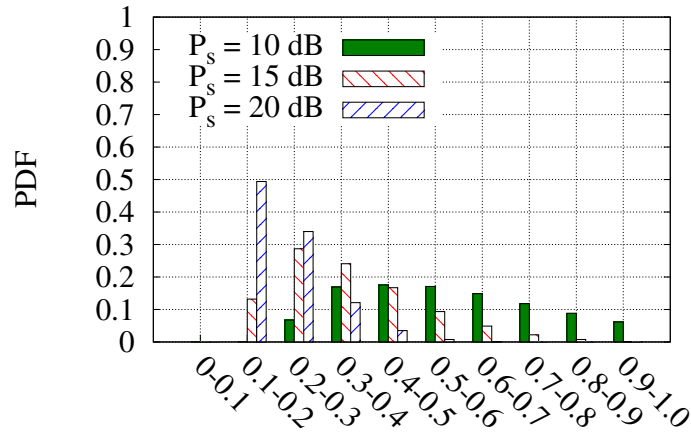

Secondary link outage probability

(b) Close scenario

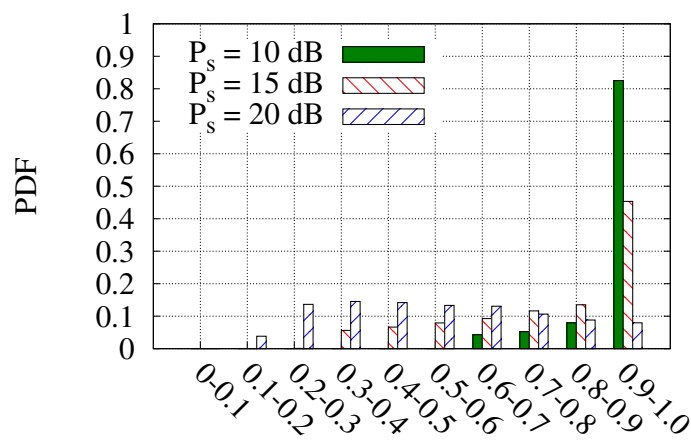

Secondary link outage probability

(d) Far scenario

Fig. 3. The Probability Density Function (PDF) for both the power splitting ratio $\alpha$ and the corresponding secondary link outage probability is presented. While simulating two network scenarios, (namely, Close and Far) and for SU power $\left(P_{s}=10,15\right.$ and $\left.20 \mathrm{~dB}\right)$.

ondary link outage probability in the Far scenario compared to the Close scenario, and thus, we expect a high useless secondary transmissions, because the power splitting ratio $\alpha$ is in the range of $[0.9-1.0]$ most of the time as shown in Figure $3 \mathrm{c}$ and the remaining amount of power $(1-\alpha) P_{s}$ for secondary communication is too little to satisfy the secondary data rate $R_{s}$. Accordingly, SUs and before start transmitting, they have not only to satisfy the condition in Equation (2) to protect the primary system, but also they have to take into account the effect on the secondary link and to satisfy the condition in Equation (3), where the secondary link outage probability has to be less than a given threshold $(\xi)$, otherwise SUs must keep Silent to avoid useless transmissions and so to save power:

$$
\begin{aligned}
& \mathbb{P}\left\{\log _{2}\left(1+\left|h_{s, s}\right|^{2}(1-\alpha) P_{s}\right)<R_{s}\right\}<\xi \\
& \stackrel{\circ}{=} 1-\exp \left(\frac{2^{R_{s}}-1}{\mu_{s s}(1-\alpha) P_{s}}\right)<\xi
\end{aligned}
$$

where $h_{s, s}$ is exponentially distributed with mean $\mu_{s s}$.

Next, we evaluate the SUs performance while applying the condition in Equation (3), i.e., keeping silent. Moreover, we compare our results to the Split protocol.

\section{PERFORMANCE EVALUATION}

The performance of our proposal, i.e., Split protocol with Power Saving (Split-PS), is measured in terms of expected throughput as it is defined in [6]. Accordingly, the expected throughput is given by:

$$
C=R\left(1-\mathbb{P}_{\text {out }}\right)
$$

where $\mathbb{P}_{\text {out }}$ is given by Equation (1).

Afterward, the percentage of SUs energy gain is presented, where SUs are going to decide either to transmit or to keep silent and avoid useless transmissions, whenever they have the chance to transmit. We use the Split protocol in [7] as reference for comparison.

\subsection{Results}

This section presents numerical results, where we consider the system model in section 2 We set SU power $P_{s}$ and PU power $P_{p}$ equal to 10 and $12 \mathrm{~dB}$, respectively. Moreover we set the SU rate $R_{s}$ and the PU rate $R_{p}$ equal to $3 \mathrm{bit} / \mathrm{s} / \mathrm{Hz}$.

Figures 4 and 5 present the expected throughput for both network scenarios that are defined in section 3 while varying the outage probability threshold for the secondary link between $[0.3-0.9]$. One can notice that our proposal with power saving Split-PS starts 


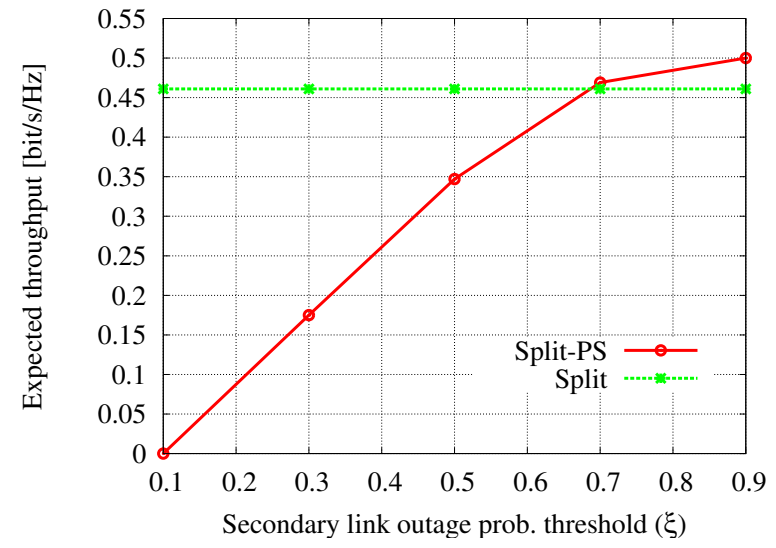

Fig. 4. The SU expected throughput is presented for the Close scenario. While the secondary link outage probability threshold $\xi$ is varying between $[0.3,0.9]$.

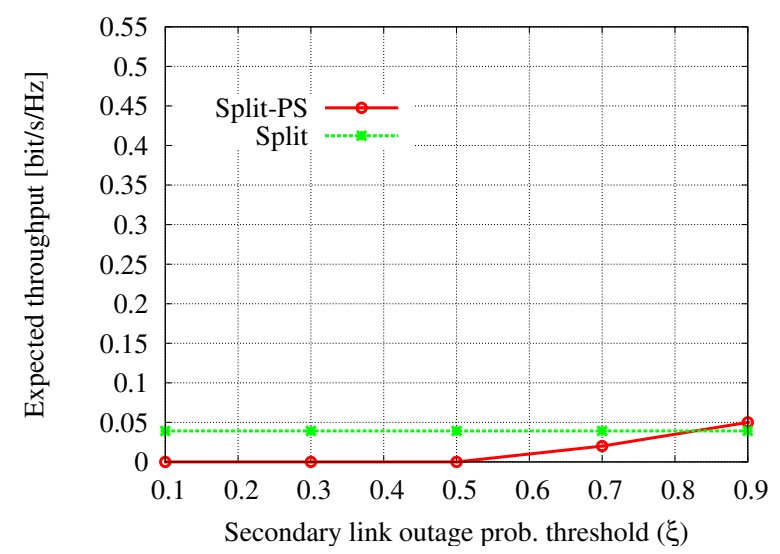

Fig. 5. The SU expected throughput is presented for the Far scenario. While the secondary link outage probability threshold $\xi$ is varying between $[0.3,0.9]$.

outperforming the Split protocol for high secondary link outage probability thresholds. Where this is true in the Close scenario for a secondary link outage probability threshold higher than 0.7 , while in the Far scenario this is true for a secondary link outage probability threshold higher than 0.8 . Moreover, for high values of outage threshold we notice the slightly higher performance of the Split-PS protocol compared to the Split protocol. Indeed, in our proposal Split-PS the common parts between the primary and secondary messages will be transmitted with full power and no need for power split operation, thanks to our proposal of utilizing the OFDM-CP on the PU transmitter.

Interestingly, from Figure 6 we can see how the SUs in Close scenario (for $\xi>0.7$ ) and in $\mathrm{Far}$ scenario (for $\xi>0.8$ ), they are able to save around $10 \%-15 \%$ and $65 \%-75 \%$ of their energy, respectively, by avoiding the useless transmissions, while still outperforming the Split protocol in terms of expected throughput, as it is confirmed by Figures 4 and 5

Next, we draw some conclusions.

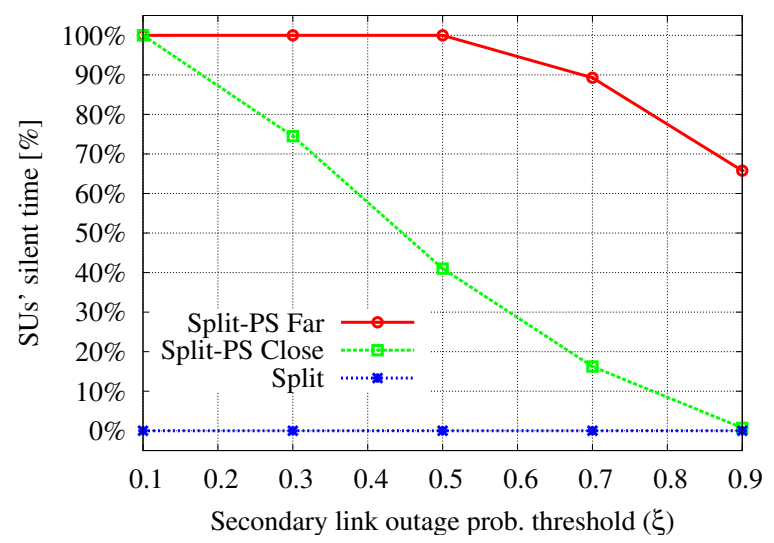

Fig. 6. The percentage of the SUs' energy gain is presented for the Split-PS protocol over the Close and Far network scenarios, respectively. While the secondary link outage probability threshold $\xi$ is varying between $[0.3,0.9]$.

\section{CONCLUSIONS}

The SU power saving in the HARQ based overlay cognitive radio access paradigm is investigated. Where the effect of the power splitting ratio on the secondary transmissions is presented and a simple power saving technique is proposed. Moreover, the utilization of the use of OFDM-CP on the PU is proposed, to allow a coherent sum between the secondary relay signal and the primary signal on the PU receiver. An extensive simulation runs were carried out to validate our results.

According to our results, a consideration for the secondary link performance while computing the power splitting ratio is very important to avoid the useless transmissions and to gain a significant amount of energy.

\section{REFERENCES}

[1] I. Maric S. Srinivasa A. Goldsmith, S. Jafar. Breaking spectrum gridlock with cognitive radios: An iinformation theoretic perspective. Proc. IEEE, 2009.

[2] P. Viswanath A. Jovicic. Cognitive radio: An information theoretic perspective. IEEE Trans. Inf. Theory, 2009.

[3] A. Goldsmith J. Sachs, I. Maric. Cognitive cellular systems within the tv spectrum. New Frontiers in Dynamic Spectrum, IEEE Symposium, 2010.

[4] S. Shama L. H. Ozarow. Information theoretic considerations for cellular mobile radio. IEEE Trans. Veh. Technol, 1994.

[5] T. Krauss M. Batariere, K. Baum. Cyclic prefix length analysis for $4 \mathrm{~g}$ ofdm systems. IEEE Vehicular Technology Conference, 2004.

[6] A. D. Wyner M. Katz, S. Shamai. Transmitting to colocated users in wireless ad hoc, sensor networks. IEEE Trans. Inf. Theory, 2005.

[7] A. Nosratinia R. Tannious. Cognitive radio protocols based on exploiting hybrid arq retransmissions. IEEE Trans. Wirel. Commun., 9:2833-2841, 2010.

[8] A. Nosratinia R. Tannious. Reducing co-existence penalty of retransmission-based cognitive radio protocol. Electronic letters, 47:409-411, 2011. 
[9] S. Srinivasa, C. J. Erkelens, and R. M. Steinman. The throughput potential of cognitive radio: A theoretical perspective. IEEE Communications Magazine, 45:73-79, 2007. 